\title{
Effect of electrical stimulation on muscle atrophy and spasticity in patients with spinal cord injury - a systematic review with meta-analysis
}

\author{
Sergio Ricardo Thomaz ${ }^{1} \cdot$ Gerson Cipriano Jr $\mathbb{D}^{2} \cdot$ Magno Ferreira Formiga $\mathbb{D}^{3} \cdot$ Emerson Fachin-Martins ${ }^{2}$. \\ Graziella França Bernardelli Cipriano ${ }^{1,2} \cdot$ Wagner Rodrigues Martins ${ }^{2} \cdot$ Lawrence Patrick Cahalin $^{3}$
}

Received: 26 April 2018 / Revised: 29 December 2018 / Accepted: 3 January 2019 / Published online: 29 January 2019

(c) International Spinal Cord Society 2019

\begin{abstract}
Study design This work is a systematic review with meta-analysis

Objective Evaluate the effect of electrical stimulation (ES) on skeletal muscle volume and spasticity in individuals with spinal cord injury (SCI).

Setting University of Brasilia, Brazil

Methods Searches were conducted of the Cochrane Library, MEDLINE, CINAHL, PEDro, PsycINFO and EMBASE electronic databases for relevant articles published up to June 2018. No restrictions were imposed regarding the year of publication. The inclusion criteria were randomized controlled trials involving adults with SCI comparing ES to an active or passive control. Two independent reviewers extracted the data from the selected studies and methodological quality was assessed using the PEDro scale.

Results The initial search led to the retrieval of 164 studies, seven of which met the eligibility criteria, but only six were included in the meta-analysis. The six studies comprised 104 patients with complete or incomplete SCI. In the two studies that investigated the use of ES on muscle volume of the lower limbs, the overall effect was statistically significant in patients with acute SCI (mean difference: 0.86 ; $95 \%$ CI: 0.04 to $1.69 ; p<0.04$ ). Among the four studies that examined the use of ES for spasticity of the lower limb, the overall effect was non-significant (mean difference: 0.55 ; 95\% CI: -0.31 to $1.41 ; p=0.21)$.

Conclusions Electrical stimulation was found to be an effective method for increasing muscle volume in SCI patients, but had no effect on spasticity. Further investigation of the effect of ES on spasticity in SCI is needed.
\end{abstract}

\section{Introduction}

Patients with spinal cord injury (SCI) experience skeletal muscle atrophy below the level of the injury, which exerts a negative impact on independence and health [1]. Reduced skeletal muscle mass due to the absence of physical activity

Sergio Ricardo Thomaz

sthomaz@yahoo.com.br

1 Health Sciences and Technologies, Department of Physical Therapy, University of Brasilia, Brasília, DF, Brazil

2 Doctoral Program in Physical Education and Rehabilitation Science, University of Brasilia, Brasilia, DF, Brazil

3 Department of Physical Therapy, University of Miami Miller School of Medicine, Coral Gables, FL, USA and voluntary contraction of paralyzed limbs in patients with complete SCI may produce secondary health complications, such as diabetes mellitus [2], abnormal glucose tolerance [3], cardiovascular disease [4] and peripheral vascular disease. Therefore, exercise plays an important role in improving fitness, physical activity and health in this population [4].

For patients with SCI who are unable to perform traditional forms of exercise, neuromuscular electrical stimulation (ES) or functional ES may be an alternative method to increase skeletal muscle mass and strength. Functional ES involves pairing ES simultaneously or intermittently with a functional task [5, 6]. Neuromuscular ES is typically provided at higher frequencies $(20-50 \mathrm{~Hz})$ to produce skeletal muscle tetany and contraction that may then carry over into functional tasks [6]. 
ES has been recommended as an effective rehabilitation intervention that can attenuate the detrimental effects of illness or injury on skeletal muscle and improve one's metabolic profile [6]. Furthermore, ES appears to be well tolerated and has been studied in regard to its effects on preventing skeletal muscle atrophy as well as improving skeletal muscle volume and function. Although the effects of ES on muscle volume in patients with SCI remains unclear [7-9], a recent meta-analysis has suggested that ES of the abdominal muscles is an effective technique for improving respiratory function in such patients [10]. The only other meta-analysis found describes the possible effects of ES on increasing bone mineral density below the level of the injury in patients with chronic SCI [11].

Spasticity is also a common complication of SCI and is clinically diagnosed through the detection of exaggerated tendon reflexes and velocity-dependent muscle hypertonia in the resting state, typically affecting the muscles surrounding the ankle, which can lead to difficulty performing functional activities [12, 13]. The Ashworth and Modified Ashworth scales are among the most common methods to assess spasticity [14, 15].

Several studies have shown that ES can diminish specific components of spasticity, including the exteroceptive flexion reflex and clonus in patients with SCI [16-18]. Furthermore, it has been suggested that ES administered to peripheral sensory nerves may reduce spasticity in patients with SCI by modulating abnormal spinal inhibitory circuits $[19,20]$. However, some authors have suggested that the reduction in spasticity immediately after ES may merely reflect muscle fatigue, which can last up to $24 \mathrm{~h}$ [21, 22].

In view of the lack of consensus regarding ES in patients with SCI, we performed a systematic review with metaanalysis to evaluate the effect of ES on skeletal muscle volume and spasticity in patients with SCI [23].

\section{Methods}

\section{Registration and setting}

This study was registered with the International Prospective Register of Systematic Reviews (CRD42017069583). The systematic review was performed using the PICO method: Population: patients with spinal cord injury; Intervention: ES; Comparison: other intervention (active control) or no intervention (passive control); Outcomes: variables related to physical function, such as muscle volume evaluated by cross-sectional area or dual energy X-ray absorptiometry and spasticity evaluated using the Ashworth scale or modified Ashworth scale. The Modified Ashworth scale is based on the perceived resistance to movement using a six-point scale (grades: $0,1,1+, 2,3,4)$, with 0 representing normal movement and 4 reflecting maximum resistance.

\section{Search methods}

The Cochrane Library, MEDLINE, CINAHL, PEDro, Embase and PsycINFO databases were searched for relevant articles published up to June 2018. Manual searches of the reference lists of the selected studies and two previous systematic reviews were also conducted [10, 11]. No restrictions were imposed in regard to language or year of publication.

The following keywords and search strategy were used for the electronic search: Spinal Cord Injuries (MESH), OR Quadriplegia (MESH), OR Paraplegic (MESH), AND Electric Stimulation Therapy (MESH), OR Functional Electrical Stimulation, OR Neuromuscular Electrical Stimulation, OR Transcutaneous Electrical Nerve Stimulation (TENS) (MESH). Based on the guidelines of the Cochrane Handbook for Systematic Reviews of Interventions [24], we did not include the "outcome" portion of the PICO strategy in order to increase the number of studies screened.

\section{Selection criteria}

The inclusion criteria were decided by consensus between the reviewers. Only randomized controlled trials of adult patients with complete or incomplete SCI were included. Studies with no follow-up and those with incomplete data were excluded. Incomplete data were determined when data were not available in the text and its extraction was not possible through graphic analyses. The authors of 7 articles from which data could not be extracted were contacted, however none of the 7 authors responded. Studies with any type of ES as an intervention for improving skeletal muscle volume and spasticity compared to other treatments, including no treatment or placebo ES, were included. Studies without a comparative group were excluded.

\section{Study selection}

After the initial search of the databases and removal of duplicates, articles were selected after reading the title, abstract and full text. The titles of articles retrieved during the search were analyzed first. The abstracts of those with potential relevance were then analyzed for the pre-selection of articles to be submitted to full-text analysis, followed by the methodological analysis of the selected studies. Divergences of opinion between the reviewers regarding the selection of studies were resolved by consensus. The reference lists of the selected articles were also reviewed to find possible additional studies. 


\section{Data extraction}

The following data were collected: study design, participants, time after spinal cord injury [divided into acute ( $2 \mathrm{~h}-6$ months), and chronic (6 months and beyond) phases] [25], interventions and outcomes including muscle volume (evaluated by cross-sectional area or dual energy X-ray absorptiometry) and spasticity (using the Ashworth scale or Modified Ashworth scale). In studies with more than one result that compared the effects of two different treatments (e.g., ES + stationary bike or ES + isometric contraction) or more than one follow-up evaluation, we utilized only the data with the greatest effect size. Data extraction were performed by a single reviewer (S.R.T.) and checked by a second reviewer (G.C.J.). Divergence of opinion was resolved by consensus.

\section{Analyses}

The data were processed following the guidelines of the Cochrane Handbook for Systematic Reviews of Interventions [24]. For continuous variables, changes in scores were compared between the intervention group (ES) and control group (active or passive) using the mean difference and 95\% confidence interval (CI) in the Ashworth or Modified Ashworth Scale score for spasticity and the standardized mean difference and $95 \%$ CI for skeletal muscle volume (different measurement methods) [24]. Heterogeneity among the studies was explored qualitatively by comparing the characteristics of the studies and quantitatively by using the chi-squared test of heterogeneity and the $I^{2}$ statistic. When appropriate, the results of the studies were combined for each outcome to determine the overall estimate of the treatment effect. The most effective model for meta-analysis was selected based on qualitative and quantitative analyses as well as the analysis of publication bias. A random-effects model was used in all analyses.

All analyses were conducted using Review Manager version 5.3.5.

\section{Appraisal of methodological quality}

The methodological quality of the selected randomized controlled trials was scored using the Brazilian Portuguese version of the PEDro scale, which has 11 items (random allocation; allocation concealment; baseline comparability; blinding of subjects; blinding of therapists; blinding of assessors; adequate follow-up; intention-to-treat-analysis; between-group comparisons; point estimates and variability) scored as "yes" or "no". As the first item is not used for the calculation, the quality score ranges from 0 to 10 points. Trials with a PEDro score of $\geq 6$ points were classified as having high quality and those with a score of $<$ 6 points were classified as having low quality.

\section{Results}

\section{Identification and selection of studies}

The initial search led to the identification of 3832 articles. After the removal of duplicates and the application of the inclusion criterion (randomized clinical trials), 164 studies were submitted to an analysis of the titles and abstracts, 31 of which were considered potentially eligible. Each of these articles were submitted to full-text analysis, resulting in seven articles which were deemed eligible and were included in the review. However, one study was not included in the meta-analysis due to an active control group [26]. Figure 1 shows the flowchart of the study selection process with reasons for the exclusion of articles.

\section{Description of studies and participants}

The six studies included in the meta-analysis comprised a total of 104 patients with incomplete or complete SCI. Descriptions of all studies are presented in Tables 1 and 2 . Two studies analyzed muscle volume [27, 28] and four analyzed spasticity [12, 14, 29, 30]. Four studies recruited patients with cervical and thoracic SCI [12, 14, 28, 30] and two studies selected patients with complete thoracic SCI $[27,29]$. The duration of time since SCI reported in the studies allowed us to examine the effects of ES on skeletal muscle volume and spasticity in patients with non-chronic versus chronic conditions. The effect of ES during the acute period was examined in studies that analyzed muscle volume and spasticity in patients with a mean of 5.68.8 weeks and $<4$ months since the injury, respectively. The effect of ES in the chronic period was examined in studies that analyzed muscle volume and spasticity in patients with a mean of 9.5 years and 11.5-114 months post-injury, respectively.

\section{Intervention}

The studies that measured skeletal muscle volume used ES with cycling resistance [28] or isometric contraction [27]. The control groups in these studies were not subjected to ES. In the study examining the effect of ES during isometric contraction, the control group was subjected to the same stimulation, but the amplitude was different $(140 \mathrm{~mA}$, intervention group; $0 \mathrm{~mA}$, control group). One study analyzed a third group [28] and we chose to use the group not subjected to any intervention in our analyses. The studies 
Fig. 1 Flow diagram of the review process

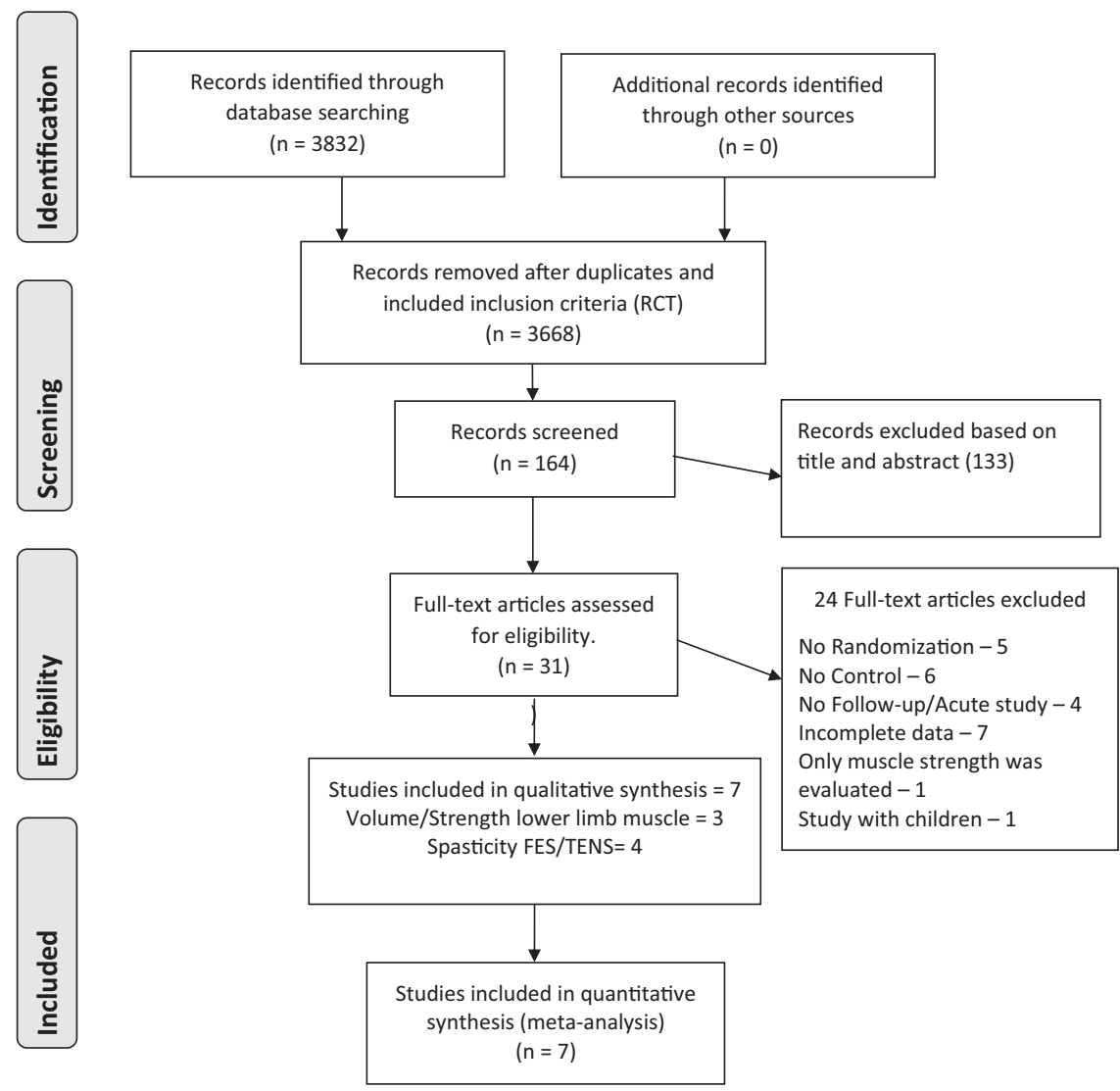

were quite heterogeneous in regard to the stimulation parameters, as shown in Tables 1 and 2.

Two electrical stimulation currents were used in the studies examining the effect of ES on spasticity. Two studies compared the effects of ES to combined physical and occupational therapy $[12,29]$. The other two studies compared ES with resistance exercise and treatment with baclofen [14, 30]. Summaries of the interventions in the different studies are presented in Tables 1 and 2.

\section{Methodological quality and risk of bias}

Table 3 displays the results of the analysis of the methodological quality of the randomized controlled trials. The mean score on the PEDro scale was 6.85 (range: 6 to 8). Given the small number of studies included in the review (less than 10), it was considered inappropriate to present publication bias using funnel plots.

\section{Effect of electrical stimulation on muscle volume}

Figure 2 shows the results from the two studies investigating skeletal muscle volume at baseline and after the acute administration of ES. These studies involved 26 patients (12 in the intervention groups and 14 in the control groups) [27, 28]. One study [27] used cross-sectional area to measure muscle volume and one [28] used dual energy X-ray absorptiometry. These studies were homogeneous $\left(\chi^{2}:=\right.$ $\left.0.04 ; \mathrm{p}=0.85 ; \mathrm{I}^{2}: 0 \%\right)$. The overall effect of ES on muscle volume of the lower limbs was statistically significant (mean difference: 0.86; 95\% CI: 0.04-1.69; $p=0.04$ ) favoring ES over the control (Fig. 2).

\section{Study not included in meta-analysis}

Despite being selected and presenting all inclusion criteria, one study [26] was not included in the meta-analysis because it was the only one that had an active control group (aerobic and resistance training). Therefore, it could not be compared to the other studies, which had a passive control group. This study compared an ES-assisted walking exercise program to aerobic and resistance training (control group) in 27 individuals with chronic incomplete motor impairment for four months. Although the intervention did not result in a change in body composition, long-term follow-up revealed that it may maintain muscle volume.

\section{Effect of electrical stimulation on spasticity}

Figure 3 shows the results of the four studies [12, 14, 29, 30] examining spasticity at baseline and after ES, which involved 


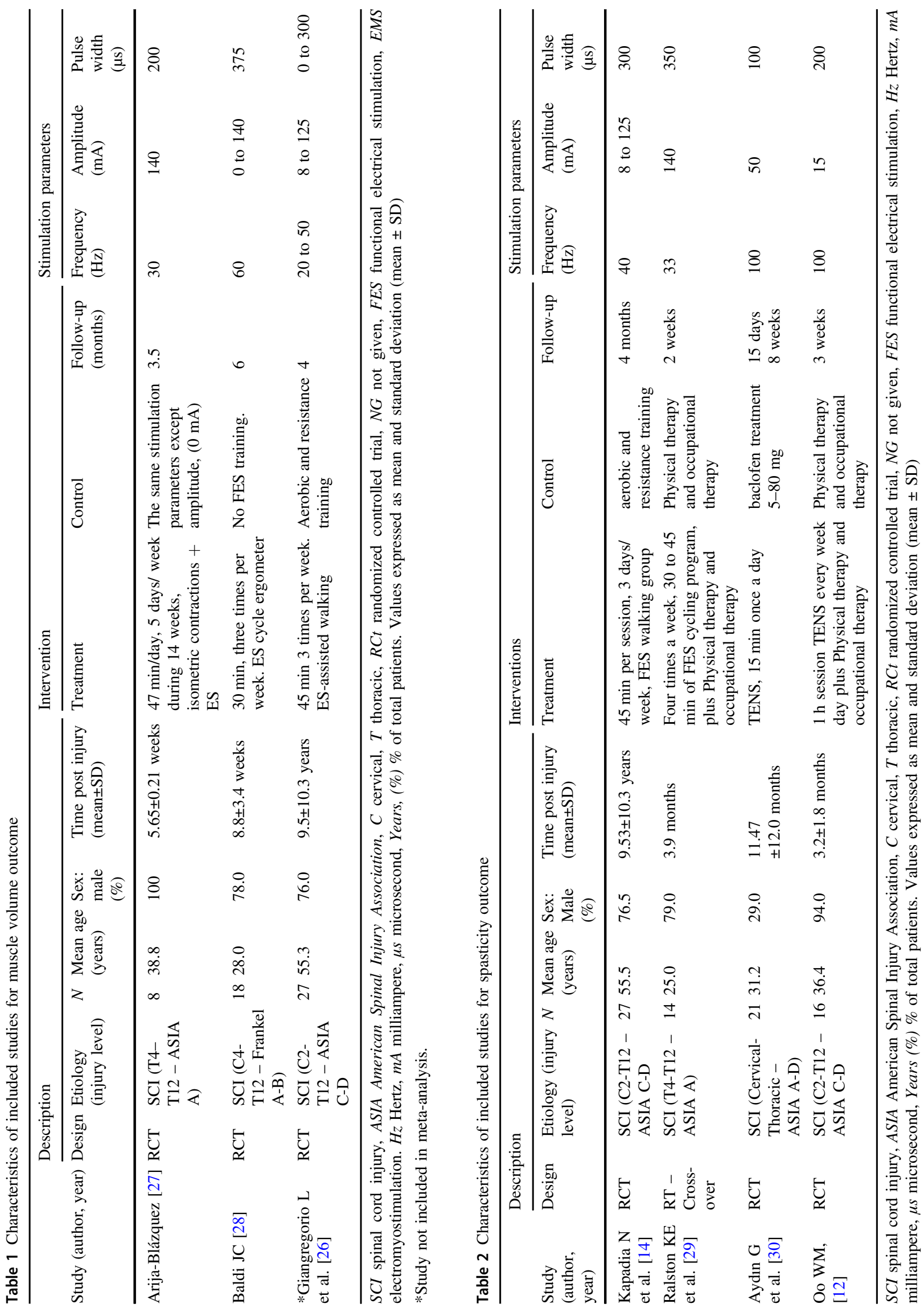




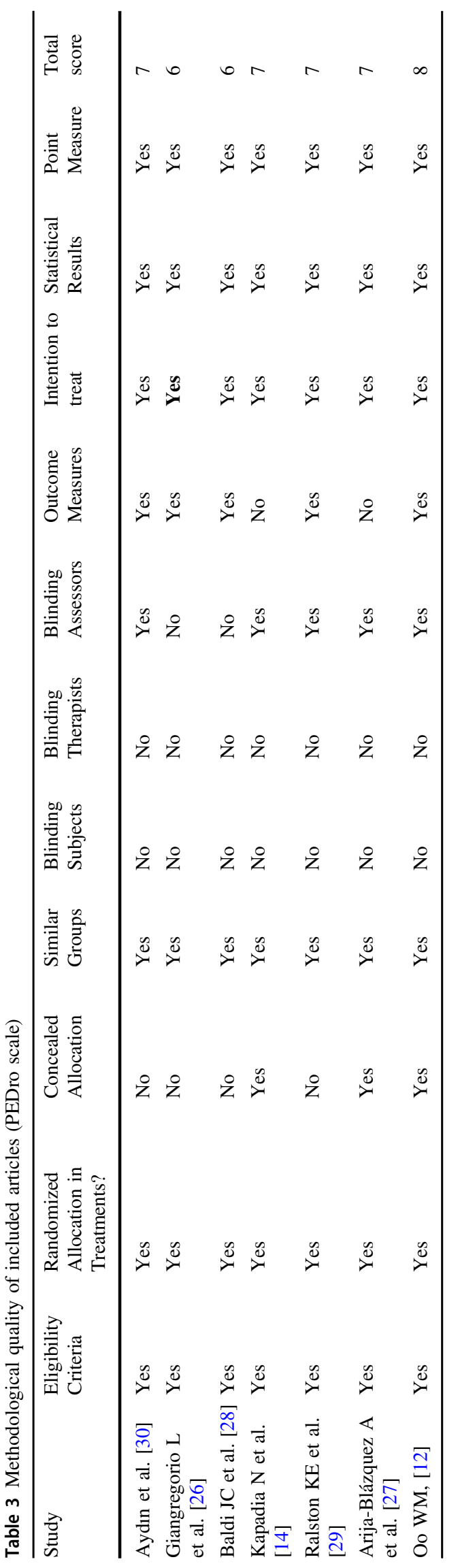

a total of 108 patients (44 in the intervention groups and 62 in the control groups). These studies were homogeneous $\left(\chi^{2}=2.21 ; p=053 ; I^{2}: 0 \%\right)$. The overall effect of ES on spasticity of the lower limbs was non-significant (mean difference: $0.55 ; 95 \% \mathrm{CI}$ : -0.31 to $1.41 ; p=0.21$ ), although three studies $[12,29,30]$ demonstrated a decrease in spasticity (Fig. 3).

\section{Discussion}

\section{Data Archiving}

Spinal cord injury is associated with skeletal muscle weakness and atrophy below the level of the injury. Physical inactivity in patients with SCI is associated with several complications, such as pressure sores, fractures, diabetes, impaired glucose tolerance, deep venous thrombosis, and cardiovascular disease [28, 30]. Another consequence of SCI is spasticity and an increase in muscle tone due to upper motor neuron lesions as well as increased tendon reflexes, which often produce abnormal joint mechanics [12, 30, 31]. Therefore, skeletal muscle atrophy and spasticity are key manifestations of SCI that require effective management to improve the prognosis and quality of life of patients.

In the present meta-analysis, the administration of ES was found to increase muscle volume, reducing lower limb atrophy in patients with SCI when compared to a control group or other form of treatment. To the best of our knowledge, this is the first meta-analysis to evaluate the effects of ES on muscle volume and spasticity in patients with SCI.

\section{Effect on muscle volume}

Increased skeletal muscle volume may facilitate greater skeletal muscle pumping, which has the potential to minimize several adverse manifestations of SCI. For example, ES cycling or ES-induced leg exercise can reduce skeletal muscle atrophy and potentially improve circulation by activating the skeletal muscle pump of the legs [32].

The significant improvement in muscle volume associated with ES is an important and clinically relevant finding that can assist in the management of patients with SCI. All studies analyzed in the present review measured lower limb muscle volume (mainly in the quadriceps muscle). The average change after intervention was similar in three studies $(14.1 \%, 10.8 \%$ and $9.3 \%)$ despite having different follow-up periods including 4, 3.5 and 6 months, respectively [26-28]. It is also important to note that the frequency and duration of ES differed between studies, with a range of three to five days/week and 30 to $60 \mathrm{~min}$ of ES 
Fig. 2 Comparison between ES versus control on muscle volume. ES electrical stimulation, CSA cross-sectional area, DEXA dual energy X-ray absorptiometry

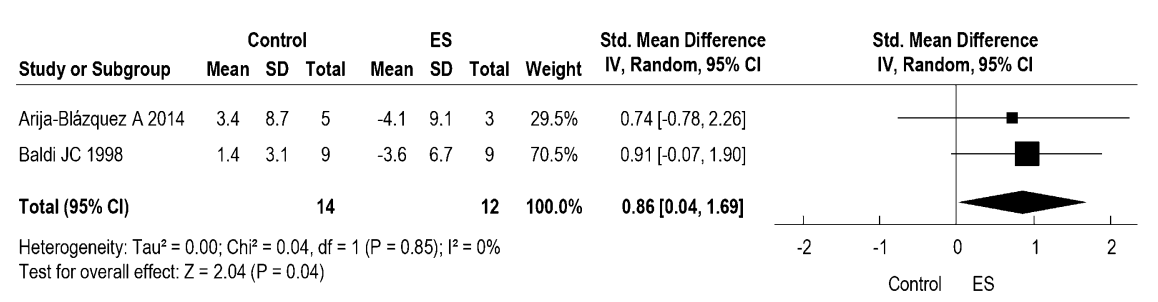

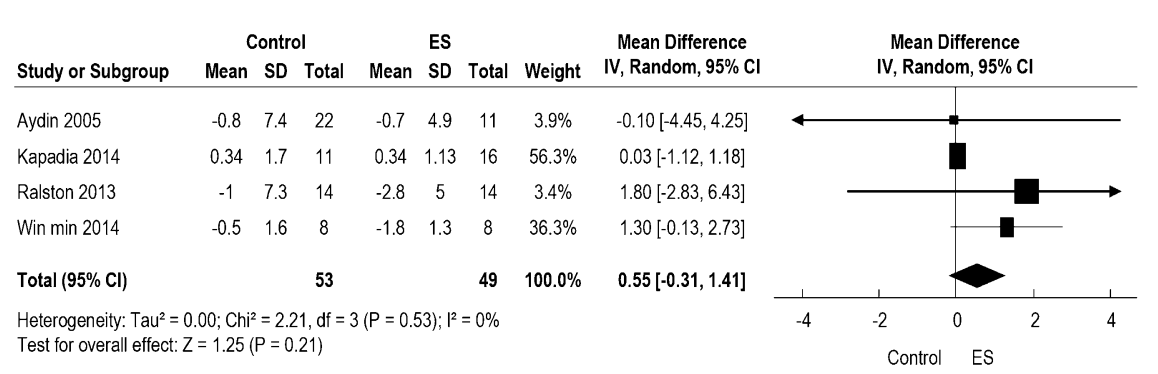

Fig. 3 Comparison between ES versus control on spasticity. ES electrical stimulation, FES functional electrical stimulation, TENS transcutaneous electrical nerve stimulation

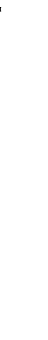

per session. In view of the above, an optimal protocol for ES after SCI is needed which may facilitate improved skeletal muscle volume.

Several studies not included in this review because they did not meet our inclusion criteria have used ES to improve skeletal muscle function in patients with SCI. Four studies examined the effects of ES in patients with quadriplegia [33-36] and were not included due to methodological limitations or the limited provision of data. It is also important to note that participants with incomplete SCI who retain some capacity for ambulation can be benefit from a walking program that combines ES and treadmill/walking training [37-40]. Therefore, in view of these results and the results of our meta-analysis, ES appears to be an important complement to the usual care provided to patients with SCI.

\section{Effect on spasticity}

Two of the studies included in our meta-analysis that examined the effects of ES on spasticity had opposite results. In the study by Kapadia [14], the use of ES-walking in 27 patients with chronic SCI led to an increase in spasticity after four months of 45-minute sessions performed three times a week. Conversely, Ralston et al [29] found that ES-cycling in 14 patients with recent SCI led to a decrease in spasticity after two weeks of 30- to-45-min sessions performed four times a week. In view of these results, the length of time since the injury, duration of stimulation and the functional task combined with stimulation appear to be important factors to consider when using ES in patients with SCI to decrease spasticity.

In our meta-analysis, we observed that spasticity decreased after a short time period (two to three weeks) in two studies with a total of the 37 patients with incomplete injuries [12, 29]. Notably, Aydin et al. [30] found that the decrease in spasticity was similar to that achieved with baclofen. It has been suggested that ES applied to peripheral sensory nerves may reduce spasticity in patients with SCI by modulating abnormal spinal inhibitory circuits $[19,20]$. As highlighted by Aydin et al [30], baclofen treatment has the capacity to reduce spasticity, but as a drug it has several potential side effects, which does not occur with ES. The study by Aydin et al also observed significant improvement in the functional disability score after repeated applications of ES, suggesting short-term efficacy [30]. Moreover, several studies have demonstrated improvements in spasticity following ES, but were not included in the present review due to study limitations (i.e., only acute effects were examined) [41-43]. Although spasticity has the potential to exert a negative influence on quality of life by restricting activities of daily living, inhibiting effective walking and requiring greater assistance from a caregiver, it has been suggested that spasticity may have several favorable characteristics in SCI such as increasing stability while sitting and standing, facilitating the performance of some activities of daily living and transfers, and increasing venous return, with the potential to decrease the incidence of deep vein thrombosis [44, 45]. Therefore, clinical decisions regarding the treatment of spasticity in patients with SCI should be made with caution and these factors should be considered. In view of our results, further investigation of the use of ES in the management of spasticity in SCI is warranted.

\section{Study limitations}

The present systematic review with meta-analysis has several limitations, such as the small number of publications eligible for inclusion and the moderate degree of methodological quality of the included studies. Furthermore, due 
to the small number of studies, we compared different ES treatments, doses and stimulation parameters as well as different groups.

Despite these limitations, the studies included in this meta-analysis were homogeneous and evaluated a respectable number of participants and we identified the need for future studies to examine the effects of ES on muscle volume and spasticity in people with SCI.

ES is effective at promoting a discrete, but significant increase in skeletal muscle volume in patients with SCI with a subsequent reduction in atrophy and an effect on muscle volume that is similar in patients with complete and incomplete injuries. In this systematic review with metaanalysis, no significant effect of ES was found on reducing spasticity in patients with SCI. Further examination of the effects of ES on skeletal muscle volume and spasticity is needed for a better understanding of the clinical usefulness of this modality in patients with non-chronic and chronic SCI.

Funding This study was funded by CAPES (88881.068134/2014-01).

Author Contributions SRT was responsible for designing the review protocol, writing the protocol and report, conducting the search, screening potentially eligible studies, extracting and analyzing data, interpreting the results, updating reference lists and creating tables. LPC conducted the meta-regression analyses, was responsible for writing the protocol and report, extracting and analyzing data, interpreting the results, updating reference lists and creating summary tables. GCJ was responsible for designing the review protocol and screening potentially eligible studies. He contributed to writing the report, extracting and analyzing data and interpreting the results MFF contributed to data extraction and provided feedback on the report. EFM provided feedback on the report. GFBC contributed to the review of the manuscript and provided feedback on the report. WRM contributed to the review of the manuscript and the meta-analysis procedures.

\section{Compliance with ethical standards}

Conflict of interest The authors declare that they have no conflict of interest.

Statement of ethics We certify that all applicable institutional and governmental regulations concerning the ethical use of human volunteers were followed during the course of this research.

Publisher's note: Springer Nature remains neutral with regard to jurisdictional claims in published maps and institutional affiliations.

\section{References}

1. Castro MJ, Apple DF Jr, Staron RS, Campos GE, Dudley GA. Influence of complete spinal cord injury on skeletal muscle within 6 mo of injury. J Appl Physiol. 1999;86:350-8.

2. Duckworth WC, et al. Glucose intolerance due to insulin resistance in patients with spinal cord injuries. Diabetes. 1980;29:906-10.
3. Bauman WA, Spungen AM. Disorders of carbohydrate and lipid metabolism in veterans with paraplegia or quadriplegia: a model of premature aging. Metabolism. 1994;43:749-56.

4. Nash MS. Exercise as a health-promoting activity following spinal cord injury. J Neurol Phys Ther. 2005;29:87-103.

5. Sheffler LR, Chae J. Neuromuscular electrical stimulation in neurorehabilitation. Muscle Nerve. 2007;35:562-90.

6. Doucet BM, Lam A, Griffin L. Neuromuscular electrical stimulation for skeletal muscle function. Yale J Biol Med. 2012;85:201-15.

7. Gorgey AS, Dolbow DR, Cifu DX, Gater DR. Neuromuscular electrical stimulation attenuates thigh skeletal muscles atrophy but not trunk muscles after spinal cord injury. J Electromyogr Kinesiol. 2013;23:977-84.

8. Needham-Shropshire BM, Broton JG, Cameron TL, Klose KJ. Improved motor function in tetraplegics following neuromuscular stimulation-assisted arm ergometry. J Spinal Cord Med. 1997;20:49-55.

9. Mazzoleni S, Stampacchia G, Gerini A, Tombini T, Carrozza MC. FES-cycling training in spinal cord injured patients. Conf Proc IEEE Eng Med Biol Soc. 2013;2013:5339-41.

10. McCaughey EJ, Borotkanics RJ, Gollee H, Folz RJ, McLachlan AJ. Abdominal functional electrical stimulation to improve respiratory function after spinal cord injury: a systematic review and meta-analysis. Spinal Cord. 2016;54:628-39.

11. Chang KV, et al. Effectiveness of bisphosphonate analogues and functional electrical stimulation on attenuating post- injury osteoporosis in spinal cord injury patients-a Systematic Review and Meta-Analysis. PLoS ONE. 2013;22:e81124. 8(11)

12. Oo WM. Efficacy of addition of transcutaneous electrical nerve stimulation to standardized physical therapy in subacute spinal spasticity: a randomized control trial. Arch Phys Med Rehabil. 2014;95:2013-20.

13. Duffell LD, Brown GL, Mirbagheri MM. Interventions to reduce spasticity and improve function in people with chronic incomplete spinal cord injury distinctions revealed by different analytical methods. Neurorehabil Neural Repair. 2015;29:566-76. (6)

14. Kapadia N, Masani K, Catharine Craven B, Giangregorio LM, Hitzig SL, Richards K, et al. A randomized trial of functional electrical stimulation for walking in incomplete spinal cord injury: effects on walking competency. J Spinal Cord Med. 2014;37:511-24.

15. Bohannon RW, Smith MB. Interrater reliability of a modified Ashworth scale of muscle spasticity. Phys Ther. 1987;67:206-7.

16. Walker JB. Modulation of spasticity: prolonged suppression of a spinal reflex by electrical stimulation. Science. 1982;9:203-4. 216 (4542)

17. Han JS, Chen XH, Yuan Y, Yan SC. Transcutaneous electrical nerve stimulation for treatment of spinal spasticity. Chin Med J (Engl). 1994;107(1):6-11.

18. Seib TP, Price R, Reyes MR, Lehmann JF. The quantitative measurement of spasticity: effect of cutaneous electrical stimulation. Arch Phys Med Rehabil. 1994;75:746-50.

19. Crone C, Nielsen J, Petersen N, Ballegaard M, Hultborn H. Disynaptic reciprocal inhibition of ankle extensors in spastic patients. Brain. 1994;117:1161-68.

20. Perez MA, Field-Fote EC, Floeter MK. Patterned sensory stimulation induces plasticity in reciprocal Ia inhibition in humans. J Neurosci. 2003;23:2014-18.

21. Daly JJ, et al. Therapeutic neural effects of electrical stimulation. IEEE Trans Rehabil Eng. 1996;4:218-30.

22. Robinson CJ, Kett NA, Bolam JM. Spasticity in spinal cord injured patients: 1. Short-term effects of surface electrical stimulation. Arch Phys Med Rehabil. 1988;69:598-604.

23. Egger M, Smith GD, O'Rourke K. Rationale, potentials, and promise of systematic reviews. In: Egger M, Smith GD, Altman DG, editors. Systematic Reviews in health care: meta-analysis in context. London, UK: BMJ Publishing Group, 2011. 
24. Higgins J, Green S. Cochrane handbook for systematic reviews of intervention. 2011. http://handbook-5-1.cochrane.org/front_page. htm. Accessed December 2018.

25. Siddiqui AM, Khazaei M, Fehlings MG. Translating mechanisms of neuroprotection, regeneration, and repair to treatment of spinal cord injury. Prog Brain Res. 2015;218:15-54.

26. Giangregorio L, et al. A randomized trial of functional electrical stimulation for walking in incomplete spinal cord injury: effects on body composition. J Spinal Cord Med. 2012;35:351-60.

27. Arija-Blázquez A, et al. Effects of electromyostimulation on muscle and bone in men with acute traumatic spinal cord injury: a randomized clinica trial. J Spinal Cord Med. 2014;37: 299-309.

28. Baldi JC, Jackson RD, Moraille R, Mysiw WJ. Muscle atrophy is prevented in patients with acute spinal cord injury using functional electricalstimulation. Spinal Cord. 1998;36:463-9.

29. Ralston KE, et al. Functional electrical stimulation cycling has no clear effect on urine output, lower limb swelling, and spasticity in people with spinal cord injury: a randomized cross-over trial. J Physiother. 2013;59:237-43.

30. Aydin G, Tomruk S, Keleş I, Demir SO, Orkun S. Transcutaneous electrical nerve stimulation versus baclofen in spasticity: clinical and electrophysiologiccomparison. Am J Phys Med Rehabil. 2005;84:584-92.

31. Carty A, McCormack K, Coughlan GF, Crowe L, Caulfield B. Alterations in body composition and spasticity following subtetanic neuromuscular electrical stimulation training in spinal cord injury. J Rehabil Res Dev. 2013;50:193-202.

32. Bakkum AJ, et al. Effects of hybrid cycling versus handcycling on wheelchair-specific fitness and physical activity in people with long-term spinal cord injury: a 16-week randomized controlled trial. Spinal Cord. 2015;53:395-401.

33. Popovic MR, et al. Functional electrical stimulation therapy of voluntary grasping versus only conventional rehabilitation for patients with subacute incomplete tetraplegia: a randomized clinical trial. Neurorehabil Neural Repair. 2011;25:433-42.

34. Popovic MR, et al. Functional electrical therapy: retraining grasping in spinal cord injury. Spinal Cord. 2006;44:143-51.

35. Kapadia NM, et al. Functional electrical stimulation therapy for grasping in traumatic incomplete spinal cord injury: randomized control trial. Artif Organs. 2011;35:212-6.

36. Harvey LA, et al. Electrical stimulation plus progressive resistance training for leg strength in spinal cord injury: a randomized controlled trial. Spinal Cord. 2010;48:570-5.

37. Field-Fote EC. Combined use of body weight support, functional electric stimulation, and treadmill training to improve walking ability in individuals with chronic incomplete spinal cord injury. Arch Phys Med Rehabil. 2001;82:818-24.

38. GranatM KeatingJF, Smith AC, Delargy M, Andrews BJ. The use of functional electrical stimulation to assist gait in patients with incomplete spinal cord injury. Disabil Rehabil. 1992;14:93-7.

39. Bobet J. Can muscle models improve FES-assisted walking after spinal cord injury? J Electromyogr Kinesiol. 1998;8:125-32.

40. Gallien P, et al. Restoration of gait by functional electrical stimulation for spinal cord injured patients. Paraplegia. 1995;33:660-4.

41. Ping Ho Chung B, Kam Kwan, Cheng B. Immediate effect of transcutaneous electrical nerve stimulation on spasticity in patients with spinal cord injury. Clin Rehabil. 2010;24:202-10.

42. Goulet C, Arsenault AB, Bourbonnais D, Laramée MT, Lepage Y. Effects of transcutaneous electrical nerve stimulation on H-reflex and spinal spasticity. Scand J Rehabil Med. 1996;28:169-76.

43. Bajd T, Gregoric M, Vodovnik L, Benko H. Electrical stimulation in treating spasticity resulting from spinal cord injury. Arch Phys Med Rehabil. 1985;66:515-17.

44. Parziale JR, Akelman E, Herz DA. Spasticity: pathophysiology and management. Orthopedics. 1993;16:801-11.

45. Adams MM, Hicks AL. Spasticity after spinal cord injury. Rev Spinal Cord. 2005;43:577-86. 